\title{
The Lost of Virtual and Simulated Identity in Online/Virtual Platform Written Adaptation of a Game "Digimon Story Cyber Sleuth: Hacker's Memory"
}

\author{
Richard Lawrence, Dr. Ribut Basuki, M.A. \\ Graduate Program, Faculty of Language and Literature, Petra Christian University \\ Surabaya, East Java, Indonesia \\ e-mail: chazzprinceton867@gmail.com, rbasuki@ petra.ac.id
}

\begin{abstract}
This creative project is a written adaptation of a video game entitled Digimon story Cyber Sleuth: Hacker's Memory (2017). This adaptation will retell a story from the aforementioned video game title about an alternate Japanese society where technology has evolved so much that people can dive into a cyber space called EDEN. By using a set of computers and a special device called Digivice. In this virtual cyberspace, a physical manifestation can be achieved by having a virtual account and many people at the beginning of the story have been using EDEN for years to do many things. The story begins with a young man who lost his virtual account. In order to get his account back he has to get involved in the world of hackers which are individuals who are able to temper the programming codes of EDEN just like how it used to be in regular computers to achieve a certain purpose. In this involvement the protagonist also encounters creatures known as Digimon which will then play a major role in the story moving forward. The protagonist also meets a hacker group called Hudie which the protagonist ends up joining in order to gain support in searching for his missing account. The theme of this adaptation is how a missing a virtual identity ends up shaping another identity for the main protagonist. This adaptation also serves as a journey for the main protagonist who at the beginning falls under the category of "hacker" which is a bad term into a "hero" at the end.
\end{abstract}

Key words: Virtual, cyber space, hacker, mental data, avatar, account, identity.

\section{INTRODUCTION}

Video game is a subject of interest to many people in the world, especially the younger generation. "Younger people were most likely to have played video games once in their life, with 67 percent of Americans aged between 18-29 years admitting to have done so" (statista.com, 2018). Keeping the data above in mind, I think that at least once in people's lifetime, they have played some form of game whether it is a soccer game on the field or maybe just a game of cards on the table. To me, games, especially video games are something to be obsessed about. The obsession toward video game is not just based on the addictive nature of video game but also "thankfully video games are draining enough to allow people to live in a virtual world for some time while forgetting about all what's going on in the real world." (Radwan, 2006-2017). Based on the quote above, I would like to point out that video game's addiction can also be a positive thing. Addiction

Thus, an addiction can also be described as craving hardly on something to the point where it can be harmful. That being said, addiction toward video games might have a positive effect. Let's say making one person the ultimate world champion of a certain game because he/she always craves playing a certain game title. In order to discuss the issues above I will use a property from one franchise that originated in Japan since 1997 which is called Digimon (Digital Monster). The anime of Digimon called Digimon Adventure (1999). Digimon Adventure tells a story about seven kids who one day got sucked into a world where creatures made of digital data exist. These creatures were later known as Digimon. Aside from the above media, the Digimon franchise has also been 
adapted to video game format. The first game was called Digimon world that was released the same year as the anime Digimon Adventure in 1999.

After exploring the brief history of games, as well as Digimon, I found myself pulled toward the Digimon franchise. Personally I have been really attracted to the creature design and the lore behind this franchise. Digimon Story Cyber Sleuth: Hacker's Memory (2017) is the most recent title that I have experienced related to the Digimon franchise. The aforementioned title came in the form of a video game for playstation vita platform. After completing the main story of that game four to five times, I think that the story element is very complex and very engaging. That being the case, I really want this type of story to be enjoyed in other ways as well so that people who do not play video game can experience this amazing story. I think it will be such a waste to not bring this story to as many media as possible. Especially because most people might just skip through the story in the video format and just enjoy the game play.

The story in Digimon Story: Cyber Sleuth: Hacker's Memory (2017) is actually about a high school student who lives in a futuristic Japan where virtual worlds exist, and people can create avatars and do things with others in the virtual world like working, gaming or just chatting. The virtual world is called EDEN. In order to explore the issues that I find interesting in the Digimon Story: Cyber Sleuth: Hacker's Memory game, I am going to make a written adaptation in the form a novel. In the spirit of making the story element of the Digimon Story: Cyber Sleuth: Hacker's Memory broader toward different types of audience, I decided that putting it in a different media entirely is the best option. "Because audiences react in different ways to different media-thanks to social and material differences" (Hutcheon, 2006) help the readers imagine the situation at one point or another in the novel.

One of the reason I select the written media is to tell the story of the above title in a significantly different way. While in the video game format, the story relies heavily on visual imagery as well as character dialog, whereas in a written format like a novel, it is possible to tell the story in a completely different way. "The telling mode (a novel) immerses us through imagination in a fictional world" (Hutcheon, 2006). Imagination is the key in experiencing a written adaptation. Instead of relying on a pre established image, I think it will be very fresh to let the readers imagine the scenarios through their own imagination because level of immersion works differently for different kinds of people. That being said, I am actually very intrigued with what the thematic element and the implied messages that Digimon Story: Cyber Sleuth: Hacker's Memory offers regarding the missing of virtual identity and the creation of a chains of events that lead to the main character becoming a whole new person. Also the protagonist's interactions with other characters in such a way kind of reflect certain aspects of addiction toward virtual and simulated identity like for example an addiction toward a virtual idol like the brand Hatsune Miku in Japan who is a virtual pop idol in real life. In order to adapt the game I will take some creative liberty in choosing the Digimon used by the protagonist as well as taking some important Digimon in the story that do not have that many character developments in the game and put my own twist into it.

Through this adaptation I would like to show how the main protagonist shapes his own identity by encountering different scenarios that do not actually concern him, which can shape his new identity and turn his virtual one into something less meaningful. Moreover, I would like to show how does the story twist the role of a "hacker" into the role of "hero" by presenting the concept of hacker as a regular job in a society filled with virtual technologies, thus hackers or computer experts are considered valuable individuals that can fix major problems in the society. 
Lawrence, Basuki: The Lost of Virtual and Simulated Identity in Online/Virtual Platform

As a way to proceed with this project I am going to mainly use the theory of adaptation by Linda Hutcheon in exploring the form that the project is going to take. In her book entitled $A$ Theory of Adaptation (2006), an adaptation is described as a repetition though in a different way or form. Part of the reason I choose adaptation as a way to bring up Digimon Story: Cyber Sleuth: Hacker's Memory is because it is a very popular way of making the title better known by people who prefer media such as books. This idea of this project also stems from how popular the concept of adaptation in this day and age.

While Digimon as a franchise is mostly geared toward children, I have encountered many people in the age of twenty one and above to enjoy it as well especially the most recent video games. Because the Digimon franchise is one big idea done in many different ways, I think that making the most recent video game which Digimon Story Cyber Sleuth: Hacker's Memory as a form of adaptation in the sense that it uses an established concept of Digimon or digital monster and apply it in a new setting. Thus, it accomplishes both nostalgia and freshness.

While in Hutcheon's book entitled A Theory of Adaptation (2006) she mentions that interacting as a medium usually employs a "goal-directed" logic (Hutcheon, 2006), which is not usually found in telling and showing medium. Keeping this idea in mind, this written adaptation will employ a more novel like tropes by using many specific descriptions for how a character looks, how a certain scene plays out, and etc. The goal-directed logic of the original video game usually involves the protagonist reaching a certain level in order to continue the main story line in Digimon Story Cyber Sleuth: Hacker's Memory. However, this written adaptation will follow a linier progression following the main character moving from point A to point B as the plot needs it. Also nodding from Hutcheon's description of what an adaptation is, this written adaptation will be told in a first person point of view unlike the original Digimon Story Cyber Sleuth: Hacker's Memory which is told in a third person point of view.

Another theory I will use is the theory of Simulation and Simulacra from Jean Baudrillard. Video game in general has always been in close relation with the concept of simulation and simulacra. While video game surely takes a lot of inspirations from the real world like Call of Duty WWII (2017) is said to take place in the battle field of the actual World War II, the fact that the game is made seventy two years after the actual World War II meant that the information put into the game must have gone through filters after filters from different parties. However, a lot of people praise the game for making the World War II setting an interesting playground while in reality; the battlefield might not even look remotely like how the game portrays it.

In another article entitled Simulation and Simulacra: A History of Video games (2017) by Andrew B.R. Elliot, there are mentions about games like the Assassin's Creed franchise that use famous historical settings and characters as its background. One of the examples can be found in Assassin's Creed IV: Black Flag (2013). Being familiar with the names of famous pirates in the past, I immediately able to point out that the setting of Assassin's Creed IV: Black Flag (2013) is during the golden era of piracy around the $18^{\text {th }}$ century.

In the story of Digimon Story: Cyber Sleuth: Hacker's Memory (2017), an element of simulation is very powerful because the whole story revolves around the search of a virtual and simulated identity which is a big problem for the protagonist and even treats it as an essential part of his whole identity. Thus, Baudrillard's theory of simulation and simulacra can be used as a way 
to explore the correlation of the characters in Digimon Story: Cyber Sleuth: Hacker's Memory (2017) with issues in the real world regarding virtual and simulated identity.

Social identity theory, though very dated, was once used on a research by Christopher J. Mckinley alongside Dana Mastro and Katie M. Warber in 2014 to identify how people of European culture that moved to America identify themselves as a group (Christopher R J. Mckinley, Dana Mastro, Katie M. Warber, 2014). Though the end result of the result is said to be a little discouraging, there is a point in this research that suggest a sense of similarity or being in the same group or in the case of that research being race, the social identity theory might just be a good way to represent how a sense of belonging to a certain group can enhance the creation of social identity. This concept is actually very tertiary in my project and acts as background detail compared to the other two. However, I think that this old theory is the one I can relate to the most when it comes to the topic of social identity.

\section{Methodology}

For this creative thesis, I'm going to do both field research and library research. The field research will be conducted by using an observation on three participants who are familiar with the franchise of Digimon. The Participants that I have chosen also have the knowledge about the game which is Digimon Story: Cyber Sleuth: Hacker's Memory (2017). The participants will include four male in their mid twenties. All four have advanced English language in their background so they will be able to understand the texts presented in the video game. In order to gain the field data, I will ask the three participants to play the game Story: Cyber Sleuth: Hacker's Memory (2017) while giving them several questions regarding the narrative structure of the game. The question will also help me find which part they think are the most interesting within the narrative structure of the video game. My first participant is a 25 years old male who has played the prequel version of Digimon Story: Cyber Sleuth: Hacker's Memory (2017). The second participant is a 26 years old male who has played a bit of the prequel game and also played a bit of Digimon Story: Cyber Sleuth: Hacker's Memory (2017). The third participant is a 24 years old male who has also played the prequel game, but has not finish Digimon Story: Cyber Sleuth: Hacker's Memory (2017). The fourth participant is a 23 years old male. He does not have exposure to the Digimon franchise as the other participant, but does play a version of its online game alongside his friends at one time.

\section{Design Concept}

The genre of this written adaptation is science fiction/fantasy. In a shorter term some may call it science fantasy. Science fantasy is described as being a story that includes both elements of science and magic. Given the element of science is usually found within the world of science fiction story while the element of magic usually found within a fantasy story, the combination of both, create what is called a science fantasy. Science fantasy is probably the least science-like product among other science fictions. For example pure science fiction such as 2001: A Space Odyssey (1968) by Stanley Kubrick which people believe to be scientifically possible. Unlike science fantasy that presents a made up technology or even adding an element of magic to the mix, science fiction usually uses technology that is close to being invented or being thought of by the society in the story. The best example of a science fantasy story would be the Star Wars franchise.

The story that I draw inspiration from is actually a Japanese animation movie entitled Summer Wars (2009). In Summer Wars (2009) there exists a slight science fiction element where the characters are able to work in a virtual space while also using the fantasy element of 
Lawrence, Basuki: The Lost of Virtual and Simulated Identity in Online/Virtual Platform

conventional monster fighting. In this creative work I will mostly use fantastical elements such as monsters and magic. The element of monster will mostly come from the background established in the Digimon franchise such as how the monster supposed to look and what they do because it is already established in the franchise.

Other than that, this creative work will also include some science fiction elements, especially the element of virtual reality. When referring to virtual reality, what I mean is how in this story people can enter the virtual world through some sort of means. One of the examples of this can be seen in The Matrix (1999). The combination of science fiction and fantasy which becomes science fantasy is going to be the genre of this creative work. By adapting the story of a preexisting video game into a written form based on the observation I do on three respondents who play the game Digimon Story: Cyber Sleuth: Hacker's Memory (2017).

\section{Synopsis}

In the world where virtual reality is a daily necessity, daily activities such as exams in school or even working can take place inside a virtual reality world called EDEN. Living in Japan as a regular high school student, the Protagonist one day loses his virtual EDEN account that just happens to contain tons of information about him which in this society is even more important than having a physical ID card. Afraid of the tons of possibilities on how people can use his account, he sets on a journey to retrieve his identity to the hacker who took it; he joined Hudie, a hacker group which job is to help people. Alongside Hudie, he discovers the existence of digimon; a digital organism that lives inside of EDEN and used by hackers as tools to do many things. While working alongside Hudie, the protagonist also becomes entangled of the Hudie member's starting from Erica, Ryuji, and also Chitose. The protagonist will be forced to help a lot of people as parts of Hudie's job and in the end making a lot of important friends. In the end once the protagonist has fallen into despair on who actually stole his EDEN account, these bonds that he formed with his digimon and his friends are the ones that lift him up. Thus, when once again his friends are challenged with the biggest problem of their lives the protagonist has to step up. In the end, the protagonist is not able to safe one of his friend because of a world changing event. However, because of that event, the real world is reset to a state where EDEN and digimon never exist as well as the one friend that the protagonist unable to save until a blue butterfly passes by him and he remembers everything from the world before the reset.

\section{Conclusion}

During the writing process of this adaptation I have encountered many difficulties. One of them is timing up the research process the eventual writing process. Because of the current condition of the world, it is quite hard for me to manage some of my participant to play the video game together in order to gain information to adapt into a written form. Also because I ended up using video calls as a mean to play together and asks them questions, it is quite difficult to ask them a more elaborate question because somehow it takes a lot of time for the participants to finish a certain chapter of the game while online. I also regret not having been able to gain firsthand experience to their reactions and emotions during the parts of the game which they might consider entertaining.

Writing this story has been such an enjoyable experience for me because the title that I adapt is so closely related to the franchise that I love so much ever since I was in kindergarten. However, I must admit that this project is quite challenging. First, turning the third person narrative of the game 
into a first person point of view is more complicated than I initially thought. At the beginning, I expect changing the point of view to be a lot easier, but it turns out that there are several points in the story where only a third person point of view can achieve so in the end I have to alter few points to fit with the written version. It is also quite challenging to describe the appearance of the digimon creatures in the non-visual media because there are so many of the in the franchise, but I think at least I describe some of them quite nicely though, I personally think that some of them need more works. I also face considerable challenge in writing the fight scene between digimons in the story. While I grew up watching the anime and reading the manga of digimon, I never actually read any of its material done in written only forms. Thus, it is quite complicated for me to think and describe the moves that a specific digimon suppose to do during each fight scenes. It is especially difficult to write the movement of the fights because I have to think of how the fight will go as well as how each character moves at the same time. However, despite all of those challenges it was still quite blast to write a digimon story.

Time management is also the biggest lesson for me because I think that I procrastinate a lot when I was writing this project. I think that I have put much time and effort in making this project; it will turn out way better than it currently is. my initial thought was that I could probably finish writing this adaption quickly because I have played the game more than five times, but when I actually started writing it, I was surprised by how many details that I have to put and where should I put them especially because of the change in point of view.

In the future I hope I can write something closely related to the franchises that I love like Gundam, super heroes, and most importantly Digimon. However, due to special circumstances of the Digimon Story Cyber Sleuth: Hacker's Memory being a video game while my work of adaptation is purely for research I will never be able to publish this work without gaining official permission from the publisher of the video game. Still I would at least like to fix all the errors that I have made so far in this written adaptation and maybe publish it to a free fan fiction website or as such and let people who prefer reading to enjoy the amazing story that the Digimon franchise have to offer.

\section{REFERENCES}

Baudrillard, J., \& Poster, M. (1988). Selected writings. Stanford, CA: Stanford University Press. Baudrillard Short Introduction. (n.d.). Retrieved September 28, 2016, from http://publish.uwo.ca/ dmann/baudrillard1.htm

Christopher R J. Mckinley, Dana Mastro, Katie M. Warber. (2014). Social Identity Theory as a Framework for Understanding the Effects of Exposure to Positive Media Images of Self and Other on Intergroup Outcomes. International Journal of Communication 8.

Deheer, Laura. (2015) Everything You Need To Know About Shonen Jump https://goodereader.com/blog/manga-and-anime-news/everything-you-need-to-know-aboutshonen-jump

Digimon.net. https://digimon.net/

Elliott, A. B. (2017). Simulations and Simulacra:. Praticas Da Historia .

Endo, Tetsuya. (2010). Digimon Xros Wars. (Anime TV Series.) Retrieved from Toei Animation.

HENDERSON, R. (n.d.). Is it Science Fiction or Science Fantasy? Retrieved from fantasy-magazine.com: http://www.fantasy-magazine.com/non-fiction/is-itscience-fiction-or-science-fantasy/ 
Lawrence, Basuki: The Lost of Virtual and Simulated Identity in Online/Virtual Platform

Giddings, S. (2007). Dionysiac Machines . The International Journal of Research into New Media Technologies , 425.

Glasser, W. (2012). PROMOTING CLIENT STRENGTH THROUGH POSITIVE ADDICTION . Canadian Journal of Counselling and Psychotherapy .

Hosoda, Mamoru. (2000). Digimon Adventure: Our War Game!. (Film.)

Retrieved from Toei Animation.

Habu, Kazumasa. (2017). Digimon Story Cyber Sleuth: Hacker's Memory. (Video Game.)

Retrieved from Media Vision.

Hamazaki, Tatsuya. (2006). Digimon Next. (Manga.)

Retrieved from Shueisha.

Hashino, Katsura. (2008). Persona 4. (Video Game.)

Retrieved from Atlus.

Hashino, Katsura. (2016). Persona 5. (Video Game.)

Retrieved from Atlus.

Hosoda, Mamoru. (2009). Sumer Wars. (Film.)

Retrieved from Warner Bros.

Hutcheon, L. (2006). A Theory of Adaptation.

Ito, Naoyuki. (2006). Digimon Savers. (Anime TV Series.)

Retrieved from Toei Animation.

Izawa, Hiroshi. (1998). Digimon Adventure V-Tamer 01. (Manga.)

Retrieved from Shueisha.

Jerz, D. G. (2000). What is Interactive Fiction?

Retrieved from jerz.setonhill.edu: https://jerz.setonhill.edu/if/intro.htm

Joeker. (2016). History of Digimon - The Franchise of many Faces

http://joekerstraightflush.blogspot.com/2016/06/Digimon-franchise-of-many-faces.html

Kakudo, Hiroyuki. (2005). Digital Monster X-Evolution. (Film.)

Retrieved from Toei Animation.

Kakudo, Hiroyuki. (1999). Digimon Adventure. (Anime TV Series.)

Retrieved from Toei Animation.

Kakudo, Hiroyuki. (2000) Digimon 02. (Anime TV Series.)

Retrieved from Toei Animation.

Kizawa,Yukio. (2001). Digimon Tamers. (Anime TV Series.)

Retrieved from Toei Animation.

Kizawa, Yukio. (2002). Digimon Frontier. (Anime TV Series.)

Retrieved from Toei Animation.

McLeod, S. A. (2008). Social identity theory https://www.simplypsychology.org/social-identity-theory.html

Medicine, A. S. (2019). Definition of Addiction.

Retrieved from asam.org: https://www.asam.org/Quality-Science/definition-of-addiction

Motonaga, Keitaro. (2015). Digimon Adventure Tri. (Film.)

Retrieved from Toei Animation.

Moody, K. L. (n.d.). What in the World is Science Fantasy. Retrieved from kaylmoody.com: https://kaylmoody.com/science-fantasy/

Nagazawa, Takao. (2002). Digimon World 3. (Video Game.)

Retrieved from BEC.

Namco, Bandai Game. (2007). Digimon World Dawn. (Video Game.)

Retrieved from BEC.

Okubo, Tetsuya. (2015). Digimon Story Cyber Sleuth. (Video Game.)

Retrieved from Media Vision. 
Peters, Megan. (2018) 'My Hero Academia' Sales Reveals Its Insane Popularity https://comicbook.com/anime/2018/03/06/my-hero-academia-popularity-manga-sales-anime/

Penn, J. (2013). Writing Fantasy: A Short Guide To The Genre. Retrieved from thecreativepenn.com: https://www.thecreativepenn.com/2013/06/27/writingfantasy/

Radwan, MSc, M.Farouk.(2006-2017) why do people,especially men, like video games https://www.2knowmyself.com/why_do_people_especially_men_like_video_games

Revoli.com https://www.revolvy.com/page/List-of-BTS-concert-tours

Romano Aja. (2018). How K-pop became a global phenomenon https://www.vox.com/culture/2018/2/16/16915672/what-is-kpop-history-explained

Sebeok, Thomas Albert. "Signs: An Introduction to Semiotics." (1994): 1-187. Web. 4 Jan. 2017.

Statista.com. (2018) Age breakdown of video game players in the United States in 2018 https://www.statista.com/statistics/189582/age-of-us-video-game-players-since-2010/

Sterling, B. (n.d.). Science Fiction Literature and Performance. Retrieved from www.britannica.com: https://www.britannica.com/art/sciencefiction/Alternative-societies

Swan-music.com. http://www.swan-music.com/history-of-j-rock/

Tester, L. (2017). Can Addiction Be Positive?

Retrieved from brainworldmagazine.com: https://brainworldmagazine.com/can-addictionpositive/

Wachowski, Lana. (1999). The Matrix. (Film.) Retrieved from Warner Bros. 